\title{
QUANTITATIVE COMPARISON OF TWO CARDIAC ELECTROPHYSIOLOGY MODELS USING PERSONALISATION TO OPTICAL AND MR DATA
}

\author{
J. Relan ${ }^{1}$, M. Sermesant ${ }^{1,3}$, H. Delingette ${ }^{1}$, M. Pop ${ }^{2}$, G. A. Wright ${ }^{2}$ and N. Ayache ${ }^{1}$ \\ ${ }^{1}$ Asclepios Research Project, INRIA, Sophia Antipolis, France \\ ${ }^{2}$ Dpt of Medical Biophysics, Sunnybrook Health Sciences Centre, Univ. of Toronto, Canada \\ ${ }^{3}$ Division of Imaging Sciences, St. Thomas Hospital, King's College London.
}

\begin{abstract}
In order to translate the important modelling work into clinical tools, the selection of the best model for a given application is crucial. In this paper, we quantitatively compare personalisation of two different cardiac electrophysiology models on the same dataset, in order to help such a selection. One is a phenomenological model, the AlievPanfilov model (1996), and the other one is a simplified ionic model, the Mitchell-Schaeffer model (2003). In the preliminary steps of model personalisation, we optimise the forward problem with the determination of an optimum time integration scheme for each model, which could result in stable and accurate simulations without the use of unnecessary expensive high temporal and spatial resolutions. Next, we personalise the two models by optimising their respective parameters, to match the depolarisation and repolarisation maps obtained ex-vivo from optical imaging of large porcine healthy heart. Last, we compare the personalisation results of the two different models.
\end{abstract}

\section{INTRODUCTION}

Quantitative and Numerical modelling of the human body has been an important research interest for the last decades, but in order to translate this work into clinical applications, there is an important need for personalisation of such models, i.e. estimation of the model parameters which best fit the simulation to the clinical data. Cardiac model personalisation is required to develop predictive models that can be used to improve therapy planning and guidance. For instance, Radiofrequency (RF) ablation therapy on patients suffering from atrial fibrillation and ventricular tachycardia has a success rate of only $50 \%$ due to non availability of clinical consensus on optimum RF ablation patterns. Thus the procedure is a trial and error process highly depending on cardiologist experience. Whereas personalised cardiac models could provide sufficiently accurate and optimum RF ablation patterns, consecutively increasing the success rate of RF therapy. To achieve this, the choice of the proper model is crucial, as the complexity and the observability of the parameters will have a huge impact on the feasibility of such personalisation. In this paper, we propose a personalisation method for cardiac electrophysiology and apply it to quantitatively compare two different cardiac electrophysiology models in terms of personalisation. This work gives insights on which model would be the most appropriate for such application. The personalisation is done using the fusion of optical and MR imaging, in order to have high quality ex-vivo data for this comparison.

The research leading to these results has received funding from the European Community's Seventh Framework Programme (FP7/2007-2013) under grant agreement $\mathrm{n} 224495$ (euHeart project).
A variety of mathematical models describing the cardiac electrical phenomenon have been developed and simulated at various scales. These models can be categorised into three main categories: Ionic models (IM), Phenomenological models (PM) and Eikonal models (EM). IM [1] characterise ionic currents flowing through the cardiac cell membrane and involve a lot of parameters and variables, thus are not well suited to solve inverse problem. However they do have analytic biological interpretation of the parameters and their influence on the behaviour of the model. EM [2] are very simple, describing only the time at which a depolarisation wave reaches a given point and does not precisely model the reaction parts of the cardiac electrical phenomenon. At the intermediate level are PM [3], which describe the action potential generation and propagation along the cell membrane, and are divided into mono-domain, modeling the transmembrane potential variable, and bi-domain modeling the intra- and extra-cellular potential variables. In this paper, we personalise two models: one phenomenological mono-domain model, the Aliev-Panfilov (AP) model [4] consisting of two nonlinear partial differential equations describing the coupled depolarisation and repolarisation processes, and one simplified ionic model, the Mitchell-Schaeffer (MS) model [5], described by two nonlinear ordinary differential equations for transmembrane potential variable and a gating variable for sodium current depicting the repolarisation phase. Authors focused recently on estimation of parameters using EM [6] and PM [7], but only on 2D surface, whereas personalisation of PM on 3D volume was studied with a simple explicit time integration scheme [8]. However, this paper is the first to present quantitative comparison of personalisation of PM and simplified IM on 3D volume. In our approach, the electrophysiology model is spatially integrated using a tetrahedral mesh of the myocardium created from MR image taking into account the fibre orientation as well, and is temporally integrated using an optimum time integration scheme.

To summarise, the main contributions of this paper are: (i) quantitative evaluation of several time integration schemes and spatial resolutions for AP and MS models, (ii) personalisation method and (iii) quantitative comparison of the personalisation of these two models using the fusion of optical and MR data.

\section{SIMULATION OF ELECTROPHYSIOLOGY MODELS}

AP Model Phenomenological mono-domain AP model is derived from the two state variables Fitzhugh-Nagumo (FHN) model. Since we simulate and observe only one single cardiac cycle, a simplified repolarisation equation is used:

$\left\{\begin{array}{l}\partial_{t} u=\operatorname{div}(D \nabla u)+k u(1-u)(u-a)-u z+J_{\text {stim }}(t) \\ \partial_{t} z=-\epsilon(k u(u-a-1)+z)\end{array}\right.$ 
$u$ is a normalised transmembrane potential, and $z$ is a variable modelling the repolarisation. The main roles of the parameters are: $k$ scales the reaction term, $a$ is a threshold for the reaction phenomenon and controls the $a p d$, and $\epsilon$ controls the coupling between the transmembrane potential and the repolarisation variable. $J_{\text {stim }}$ is the stimulation current, at the pacing location.

MS Model MS model is a simplified ionic model derived from the Fenton Karma (FK) ionic model [5]:

$$
\left\{\begin{aligned}
\partial_{t} u & =\operatorname{div}(D \nabla u)+\frac{z u^{2}(1-u)}{\zeta_{\text {in }}}-\frac{u}{\zeta_{\text {out }}}+J_{\text {stim }}(t) \\
\partial_{t} z & =\left\{\begin{array}{lll}
\frac{(1-z)}{\zeta_{\text {open }}} & \text { if } \quad z<z_{\text {gate }} \\
\frac{-z}{\zeta_{\text {close }}} & \text { if } z>z_{\text {gate }}
\end{array}\right.
\end{aligned}\right.
$$

$u$ is still a normalised transmembrane potential, and $z$ is the gating variable for sodium ion influx which depicts the repolarisation phase. $J_{i n}=\left(z u^{2}(1-u)\right) / \zeta_{\text {in }}$ represents the combination of inward sodium current which raises the action potential voltage and $J_{\text {out }}=-u / \zeta_{\text {out }}$ represents the outward potassium current that decreases the action potential voltage describing repolarisation. The parameters $\zeta_{\text {open }}$ and $\zeta_{\text {close }}$ control the repolarisation variable, with $\zeta_{\text {close }}$ directly related to the $a p d$.

The diffusion term in both models is controlled by the diffusion tensor $D$. This spatial diffusion can be related to a pseudoconductivity. In the longitudinal direction of the fibre, this pseudoconductivity is set to $d$ which is one of the parameters we adjust, and to $d / 2.5^{2}$ in the transverse directions. These electrophysiology models are solved spatially over a volumetric tetrahedral mesh of the left and right ventricles using the finite elements method, and in time using a time integration scheme, described next.

Time Integration A variety of explicit, semi-implicit and implicit schemes categorised as first, second and third order schemes have been evaluated for cardiac bidomain model [9]. Here we implement and evaluate these schemes for monodomain AP and MS model in terms of solution accuracy, stability, and computational time. A reference solution approximately representing the exact solution of the model is computed using finer 3D mesh resolution (mean edge length of tetrahedra) $h$ and temporal resolution $\delta t$ ( $h=0.33 \mathrm{~mm}$ and $\delta t=10^{-6} \mathrm{~s}$ ), with implicit scheme. Stability of a given time integration scheme is determined by varying the model parameter controlling the wave speed, and observing the solution for oscillations, for a range of $h$ and $\delta t$. Second order schemes are observed to be more stable at higher wave speed and large time steps. Whereas accuracy is determined with constant model parameters and by computing the wave speed error (as shown in Fig.1, similar for MS model) with respect to the reference solution for a range of $h$ and $\delta t$. And we observe that for small $h$ and small $\delta t$, all time integration schemes are comparable, for small $h$ and large $\delta t$ higher than first order schemes provide relative wave speed error $<1 \%$ and for large $h$ the wave speed error is high irrespective of time integration scheme used. Computational time (2.16 GHz, dual core, $2.0 \mathrm{GiB}$ ) of one time step for explicit and semi-implicit schemes are comparable and is relative to the mesh size (for $\approx 247250$ number of tetrahedra) $\approx 1 s$ and for fully implicit schemes $\approx 1 s$ for small $\delta t$ to $\approx 3 s$ for large $\delta t$. From all of this analysis, we determine an optimum $h$, time integration scheme and $\delta t$ for both models. By optimum value, we mean the largest possible value for which the relative error $<10 \%$. For AP model the optimum time integration scheme is Second order semi-implicit
Backward Differentiation (SBDF) with $\delta t=1 \mathrm{~ms}$, and for MS model it is Modified Crank-Nicolson/Adams-Bashforth (MCNAB) (second order scheme) with $\delta t=0.1 \mathrm{~ms}$, for both the models optimum $h=1.5 \mathrm{~mm}$ with one time step computation time $\approx 1 \mathrm{~s}$. SBDF is given as follows: $\left(3 / 2 u^{n+1}-2 u^{n}+1 / 2 u^{n-1}\right) / \delta t=$ $D\left(u^{n+1}\right)+2 F\left(u^{n}, z^{n}\right)-F\left(u^{n-1}, z^{n-1}\right)$. MCNAB is given as: $\left(u^{n+1}-u^{n}\right) / \delta t=9 / 16 D\left(u^{n+1}\right)+3 / 8 D\left(u^{n}\right)+1 / 16 D\left(u^{n-1}\right)+$ $3 / 2 F\left(u^{n}, z^{n}\right)-1 / 2 F\left(u^{n-1}, z^{n-1}\right)$, where $D$ represents the diffusion term and $F$ represents the reaction term of the model and $n$ is the current iteration number.
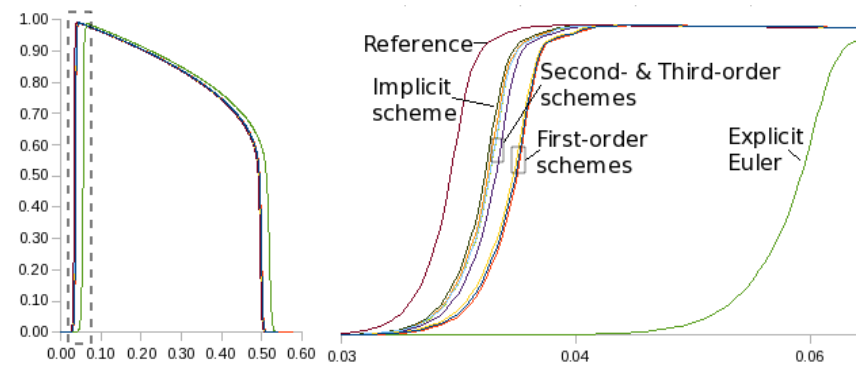

Fig. 1. Transmembrane potential $u$ wave for AP model and zoom (dashed box), temporally integrated with different schemes, on $h=$ $1.5 \mathrm{~mm}, \delta t=1 \mathrm{~ms}$ in comparison with reference (abscissa: normalised potential vs time $(s)$ ).

\section{OPTICAL AND MR IMAGE DATA PROCESSING}

In this paper we performed the adjustments using optical recordings obtained on a healthy porcine heart. The explanted hearts were attached to a Langendorff perfusion system which permits to maintain the electrophysiological integrity of the hearts over 1-2 hours. The

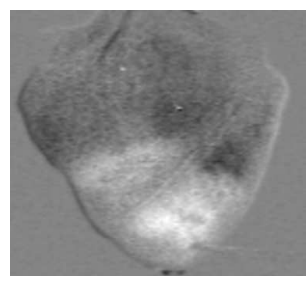

(a)

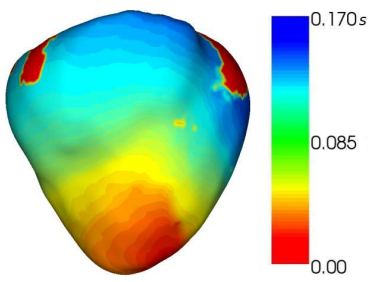

(b)
Fig. 2. (a) Raw optical signal (anterolateral view) showing action potential wave(white) and (b) Volumetric myocardial mesh generated from MR data, with projected depolarisation time isochrones (in $s$ ) derived from filtered optical data.

fluorescence dye (reflecting directly the changes of transmembrane potential) and the electro-mechanical uncoupler were injected into the perfusion line (more details are given in [10]). The hearts were paced with an electrode near the apex for $5 \mathrm{~ms}$. The fluorescence signals are captured with high temporal $(270 \mathrm{fps})$ and spatial $(<1 \mathrm{~mm})$ resolution, using a pair of CCD cameras (BrainVision Jp). At the completion of the optical experiment, the hearts were imaged using MRI for anatomy. A volumetric mesh was generated from the images with the INRIA softwares CGAL and GHS3D, resulting in a tetrahedral geometry. Diffusion Tensor Imaging was also used to estimate the fiber directions. The optical images recorded by the 2 CCD cameras were reconstructed into a 3D stereoscopic surface of the heart. Several opaque markers were glued onto the epicardium 
to provide a way to register the optical images with the surface of the model generated from MR images. We estimated a rigid transformation between the optical and MR markers by solving the least-square differences. We then projected the isochronal maps onto the registered volumetric mesh from MR Imaging with an interpolation from the triangular stereoscopic surface.

\section{MODEL PERSONALISATION METHOD}

Determination of model parameters that result in a simulation which is similar to the measured data is defined as personalisation. Here, we match the depolarisation and repolarisation time isochrones derived from the optical data to those obtained from model simulation by optimising two model parameters. This adjustment is done in two phases: Calibration and Iterative Adjustment.

Calibration. This step is used to initialise the model parameter values using analytical relationships between the measure and parameter, which are extracted by performing several model simulations for a range of parameter values and observing the corresponding measure ( $c$ or apd resp.). Then a function is fitted in the least squares sense to these values of measure.

Iterative Adjustment. This step is used to optimise the parameters with calibration result as initial guess. In order to keep computations reasonable, we divide the left ventricle into 17 zones as defined by American Heart Association and a similar division of 9 zones for the right ventricle, when an iterative adjustment is performed. The algorithm used here is a trust region method [11] which finds the minimum of a subproblem, such as a quadratic model created using gradient and approximate hessian matrix at the current search point, and which is implemented using the Trilinos solver package. Here we use an objective function that minimises the difference between the simulated and measured $c$ (or apd resp.) by iteratively adjusting the parameter value for each zone. By assuming that $c$ and apd of a zone are not strongly influenced by the neighbouring zones, the parameter value of all zones can be adjusted simultaneously, thus considerably reducing the number of simulations required.

AP Model Parameters. One dimensional analysis of the AP model [3] provides a relationship between the conduction velocity $c$ and parameters of first equation of the model, $c=\sqrt{2 k d}(0.5-a)$, the same analysis provides the relationship between parameter $a$ and action potential duration apd as apd $=(a-1)^{2} / 4 a$. It is observed that one of the parameter affecting the conduction velocity $c$ is $a$, which also controls action potential duration $a p d$, thus due to this coupling, we first adjust the parameter $a$ to the measured $a p d$, since it does not depend on any other parameter except $a$. Other two parameters affecting the conduction velocity are $d$ and $k, d$ represents the diffusion properties of the myocardial tissue and $k$ stands for the reaction term along the membrane, both constitutively represent the electrical wave propagation speed. Since we have only one measure $c$ available from the measured data, we adjust regionally (per zone) the parameter $d$ which represents the pseudo-conductivity, while keeping $k$ constant globally (value taken from the literature). The relationships between measures and parameters are only true in one dimension, as in three dimensional propagation the curvature of the diffusion wavefront affects $c$ and coupled $a p d$. Therefore we perform the calibration step of extraction of analytical relationship in 3D using the function determined from 1D analysis as given: $c(d)=\alpha \sqrt{d}+\beta$ and $a p d(a)=\left(\alpha a^{2}+\beta a+\gamma\right) / a$. These functions are used to fit in least squares sense to the measures. The constant $\beta$ is added to the relationship between $c$ and $d$ to better fit the numerical simulations and take into account the numerical diffusion and discretization errors. Once the relationship is estimated, it is used to determine the parameter value $d_{i}$ (resp. $a_{i}$ ) for the measured median measure $\widetilde{c_{i}}$ (resp. $\widetilde{a p d}$ ) for each zone $i$. For $a p d$, as we have two solutions we select the one which lies in the range of acceptable values of parameter $a$ from the literature. These paramater values of $d$ and $a$ are used then to initialise the iterative adjustment step, which minimises the following criteria on each zone: $J\left(d_{i}\right)=\left(\widetilde{c_{i}}-\widehat{c}\left(d_{i}\right)\right)^{2}$ and $J\left(a_{i}\right)=\left(\widehat{a p d}_{i}-\widehat{\operatorname{apd}}\left(a_{i}\right)\right)^{2}$, where $\widehat{c}\left(d_{i}\right)$ is the median conduction velocity over zone $i$ for the parameter $d_{i}\left(\widehat{a p d}\left(a_{i}\right)\right.$ alike).

The personalisation approach described above is defined as regional estimation (as performed per zone). Whereas another approach for apd personalisation adapted here is local estimation, where we directly use the calibration function to estimate the parameter $a$ locally at each vertex. This could be performed as apd of the measured data is more spatially independent. For the diffusion term, the iterative adjustment is needed in order to correct errors coming from spatial effects, like curvature. Also the conduction velocity estimation from the data is noisy, such regional approach allows to smooth this effect.

MS Model Parameters. The diffusion term in both models is the same, whereas the reaction terms differ. And as discussed earlier, we choose to adjust diffusion parameter $d$ to match the conduction velocity $c$. Thus the same calibration function as for AP model is used to calibrate and then we perform iterative adjustment. Whereas the action potential duration for single heart cycle is defined by the model [5] as follows: apd $=\zeta_{\text {close }} \ln \left(1 / h_{\text {min }}\right)$ where $h_{\text {min }}=4 \zeta_{\text {in }} / \zeta_{\text {out }}$. Again as we only have one measure apd available from the measured data, we choose to adjust $\zeta_{\text {close }}$, while keeping the other parameter values from the literature. It is defined by the model that $c$ has no relationship with $\zeta_{\text {close }}$, which provides no coupling between the action potential duration and the conduction velocity. Thus we can simultaneously adjust parameter $d$ and $\zeta_{\text {close }}$. The defined relationship between $\zeta_{\text {close }}$ and apd remains valid also in 3D thus allowing us to directly estimate locally at each vertex, the parameter $\zeta_{\text {close }}$ without calibration and iterative adjustment.

\section{RESULTS}

Before personalisation of both models, the error maps for action potential duration and depolarisation times are computed using a simulation with the parameter values given from literature.

Action Potential Duration. For AP model, the mean absolute error on apd before personalisation is $159.5 \mathrm{~ms}(\approx 40 \%$ of $a p d)$, reduced to $17.81 \mathrm{~ms}(\approx 5 \%)$ after personalisation with regional estimation and to $22.1 \mathrm{~ms}(\approx 6 \%)$ with local estimation of the parameter $a$. Whereas for MS model, before personalisation it is $86.99 \mathrm{~ms}$ $(\approx 21 \%)$, reduced to $8.72 \mathrm{~ms}(\approx 2 \%)$ with direct estimation of the parameter $\zeta_{\text {close }}$ locally, as shown in Fig.3.

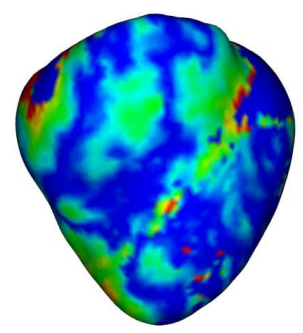

(a)

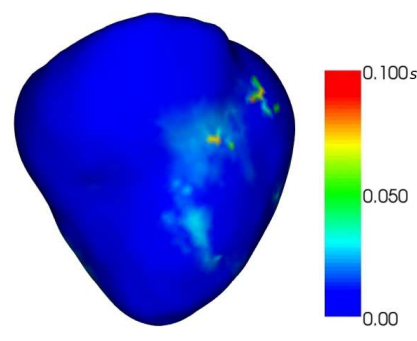

(b)
Fig. 3. Action potential duration error maps (in $s$ ) after personalisation for (a) AP model (with local estimation) and (b) MS model 


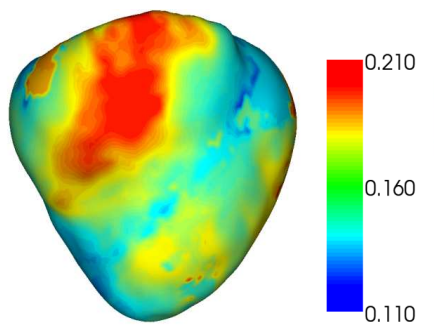

(a)

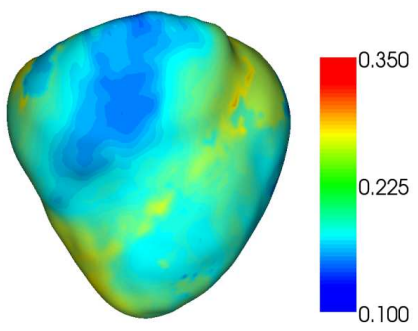

(c)
Fig. 4. Maps of (a) parameter $a$ for AP model (with local estimation) and (b) parameter $\zeta_{\text {close }}$ for MS model

Depolarisation time. For AP model, before personalisation mean absolute error on the depolarisation time is $55.77 m s(\approx 28 \%$ of depolarisation duration), to $6.2 m s(\approx 3 \%)$ with adjustment of the parameter $d$. Whereas for MS model, before personalisation error is $58.9 \mathrm{~ms}(\approx 30 \%)$, to $5.1 \mathrm{~ms}(\approx 2 \%)$ with personalisation.

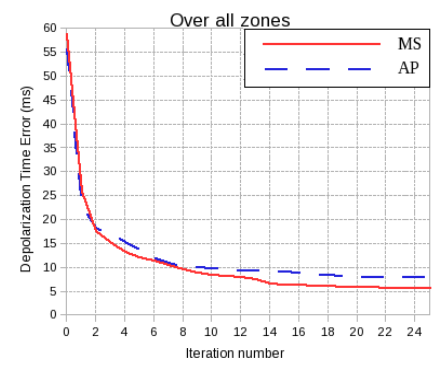

(a)

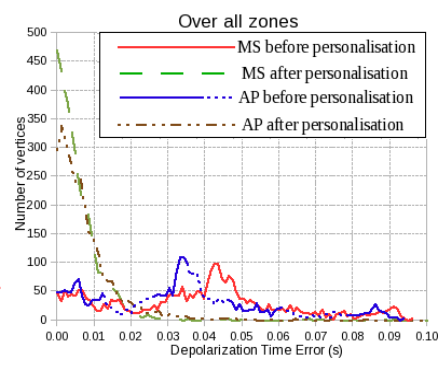

(b)
Fig. 5. (a) Depolarisation time error descent and (b) histogram.

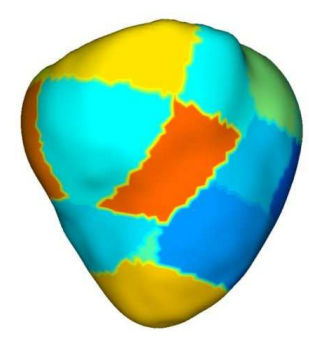

(a)

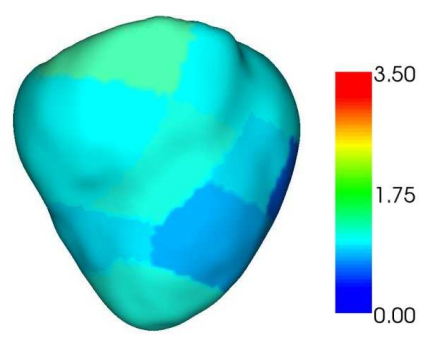

(b)
Fig. 6. Maps of parameter $d$ for (a) AP model and (b) MS model

\section{DISCUSSION AND CONCLUSION}

In this work, we have compared the personalisation of AP and MS models on the same dataset. Both models have a few number of parameters to be adjusted, thus are comparable in terms of personalisation. However, from the personalisation results, the MS model has following pros: (i) It does not have a coupling between action potential duration and conduction velocity present, whereas for AP model apd and $c$ are coupled as they both share the relationship with the parameter $a$. (ii) In terms of apd personalisation, AP model with local estimation results in higher final error with continuous parameter $a$ map, while with regional estimation which requires iterative adjustment has lower final error but discontinuous parameter $a$ map. And for the MS model, which has a defined relationship for parameter $\zeta_{\text {close }}$ that can be used locally, the error reduces only with direct estimation to considerably lower final error as shown in Fig.3, and has continuous parameter $\zeta_{\text {close }}$ map too as shown in Fig.4 (Note: apd is inversely proportional to $a$ and directly proportional to $\zeta_{\text {close }}$ ), both model parameters confirm shorter apd for right ventricle compared to left ventricle, which is a known phenomenon. (iii) Regarding depolarisation time isochrones personalisation, the MS model is more sensitive to the parameter $d$ thus resulting in sharper error descent with iterative adjustment for a few number of iterations and lower final error, while the AP model needs more iterations and has higher final error as shown in Fig.5, $(i v)$ also the parameter $d$ representing apparent conductivity in the heart is almost constant for the MS model compared to AP as shown in Fig.6, which is expected in this case of healthy heart. $(v)$ MS model has clearly defined and analytical, biological interpretation of the parameters compared to AP. On the other hand, the con for MS model is: it has a critical timestep for semi-implicit scheme 10 times smaller than the critical timestep for AP, thus making AP simulations faster. These comparison results show how such a framework can help in choosing the right model for a given clinical application, where the optical data can be replaced by endocardial Electro-Anatomical mapping of the patient. However, for further evaluation, model prediction of depolarisation and repolarisation time isochrones for different pacing locations using estimated parameter values should be compared for both models.

\section{REFERENCES}

[1] D. Noble, "A modification of the Hodgkin-Huxley equations applicable to Purkinje fibre action and pace-maker potentials," Physiology, vol. 160, pp. 317-352, 1962.

[2] M. Sermesant et al., "An anisotropic multi-front fast marching method for real-time simulation of cardiac electrophysiology," in FIMH'07, 7-9 June 2007, vol. 4466 of LNCS, pp. 160-169.

[3] J. Keener, J. Sneyd, Mathematical Physiology, Springer, 1998.

[4] R. Aliev, A. Panfilov, "A simple two-variable model of cardiac excitation," Chaos, Solitons \& Fractals, 7(3), 1996.

[5] C C. Mitchell, D G. Schaeffer, "A two current model for the dynamics of cardiac membrane," B. of Math. Biology, 65, 2003.

[6] P Chinchapatnam et al., "Model-Based Imaging of Cardiac Apparent Conductivity and Local Conduction Velocity for Diagnosis and Planning of Therapy," IEEE Transactions on Medical Imaging, 27(11):1631-1642, 2008.

[7] V. Moreau-Villéger et al., "Building maps of local apparent conductivity of the epicardium with a 2-D electrophysiological model of the heart," IEEE Trans. on Biomedical Eng. 53(8):1457-1466, 2006

[8] D. Lepiller et al., "Cardiac electrophysiology model adjustment using fusion of $\mathrm{mr}$ and optical imaging.," MICCAI'08, vol. 5241 of LNCS, pp. 678-685, 2008.

[9] M. Ethier and Y. Bourgault, "Semi-implicit time discretization schemes for the bidomain model," SIAM J. NUMER. ANAL., vol. 46, no. 5, pp. 2443-2468, 2008.

[10] M Pop et al., "Fusion of optical imaging and mri for the evaluation and adjustment of macroscopic models of cardiac electrophysiology: A feasibility study," Med Image Anal, Jul 2008.

[11] N.I.M. Gould A.R. Conn and Ph.L. Toint, Trust Region Methods, SIAM, 2000. 